\title{
Is reduction in the risk of vision loss the only benefit of photodynamic therapy in predominantly classic subfoveal choroidal neovascularization?
}

\author{
Nicola G Ghazi \\ Brian P Conway \\ James S Tiedeman \\ Steven J Yoon \\ University of Virginia Health System, \\ Department of Ophthalmology, \\ Charlottesville, VA, USA
}

\begin{abstract}
Purpose: To emphasize the effect of photodynamic therapy (PDT) on the size and progression of the neovascular lesion (NL) and evolution of the disciform scar (DS) in predominantly classic subfoveal choroidal neovascularization (SFCNV).

Methods: A retrospective study of 62 eyes treated with PDT for SFCNV was performed. The greatest linear dimension (GLD) before and at last follow-up after treatment and the size of the DS post-PDT were analyzed. A subgroup of patients with DS in their fellow eye at presentation without prior PDT was also studied. The size of the scar in these eyes was compared to that following PDT.

Results: After an average follow-up at 9 months, the size of the NL was stabilized or reduced in $64 \%$ of the study eyes with absence of fluorescein leakage in $45 \%$. Only 3 eyes (5\%) developed DS. At presentation, 14 patients already had DS in their fellow eye, the size of which was significantly larger than that post-PDT $(p=0.044)$. It was also significantly larger than that of the potential scar in the study eyes of the same subgroup of patients $(p=0.002)$ and of the rest of the patients $(\mathrm{p}=0.0001)$.

Conclusion: This study demonstrates a beneficial effect for PDT on the size of the NL and DS in SFCNV, which might be of great significance, particularly when PDT fails to prevent severe vision loss.
\end{abstract}

Keywords: age-related macular degeneration, choroidal neovascularization, classic choroidal neovascularization, disciform scarring, fluorescein angiography, photodynamic therapy

\section{Introduction}

Age-related macular degeneration (AMD) is the leading cause of legal blindness in the elderly (Klein et al 1995). The main reason for visual loss in these patients is the development of choroidal neovascularization (CNV) in the macular area, mainly in the subfoveal region (Ferris et al 1984; MPSG 1993). Subfoveal choroidal neovascularization (SFCNV) is also a common cause of vision loss in pathologic myopia (Soubrane and Coscas 2001) and the presumed ocular histoplasmosis syndrome (POHS) (Olk et al 1984). Since its introduction, PDT has had a great impact on the management of SFCNV (Bressler and Bressler 2000; Margherio 2000) and its benefit in reducing the risk of vision loss has been documented in large multicenter clinical trials in the case of AMD (TAP 1999; Bressler 2001, 2002; VIP 2001b) and myopia (VIP 2001a). Some evidence for its benefit in other causes of SFCNV is also available (Sickenberg et al 2000; Saperstein et al 2002; Busquets et al 2003). Although these trials included data regarding the fluorescein angiographic outcomes and progression of the CNV lesion following PDT, the main emphasis was on visual outcome and the reduction in the risk of vision loss. However, these studies, and data from other studies (Schmidt-Erfurth 1999; Michels et al 2000), suggest that PDT could limit 
progression of and reduce fluorescein leakage from CNV lesions. Our study exclusively discusses the beneficial effect of PDT on the progression and size of the CNV lesion, and on the evolution of the disciform scar (DS) in patients with predominantly classic SFCNV. We also comment on why this effect may be desirable even when PDT fails to reduce the risk of vision loss.

\section{Methods}

Following Institute Review Board approval, we performed a retrospective chart review of all patients who received verteporfin-mediated PDT for SFCNV at the Department of Ophthalmology at the University of Virginia Health System during a 2-year period. All patients were treated by two of us (JST and BPC). Patients with AMD and non-AMD-related predominantly classic (SFCNV) (area of classic CNV occupies $50 \%$ or more of the area of the entire lesion) were included. All cases that demonstrated ophthalmoscopic and fluorescein angiographic evidence of "retinal angiomatous proliferation" (RAP) (Yannuzzi 2001) were excluded.

To be included in this study, each treated eye had to complete at least 6 months of follow-up after the first PDT treatment. In addition, the CNV lesion had to meet the eligibility criteria set in the TAP and VIP trials (TAP 1999; VIP 2001a). Also, the treatment, re-treatment and follow-up methods had to adhere to the standard protocol of verteprofin-mediated PDT used in these trials. Only two minor deviations were allowed. First, eyes with initial best-corrected visual acuity worse than 20/200 for AMD and 20/100 for myopia were included. Second, only rarely eyes whose follow up did not precisely adhere to the TAP (1999) and VIP (2001a) follow-up protocol were included. The breach to the protocol in such eyes was that fluorescein angiography (FA) was not necessarily performed on all follow-up visits. These were stable eyes that had dramatic and sustained improvement in symptoms with visual stabilization following PDT, in which the treating physician found no funduscopic evidence of active $\mathrm{CNV}$ on later follow up visits.

In each case, the etiology of the neovascular lesion, the indication for PDT, the number of treatment sessions, and the range of follow up were noted. Also, the size of the neovascular lesion, estimated by the greatest linear dimension (GLD) of the lesion (TAP 1999), was measured before treatment and at the last follow up following treatment. Then, the percentage change in lesion size; the percentage of eyes with stable, reduced, or progressed neovascular lesion (according to TAP grading criteria of fluorescein angiographic assessment at follow up); and the percentage of eyes that developed disciform scarring following PDT were calculated.

A subgroup of 14 cases that received PDT to one eye only (study eye) and already had a DS in the fellow eye at presentation was analyzed. None of the latter eyes received PDT prior to scar development. The size of the DS, as estimated by its GLD, was measured in each case from funduscopic and angiographic data. The average size of these scars was calculated and compared to that of the DS that developed following PDT in some eyes. Also it was compared to the average size of the DS that could have potentially developed following PDT in the study eyes of the same 14 patients, and in the study eyes of the rest of the patients. In each of these comparisons, a double-tailed student's t-test was performed to assess significance.

The size of the DS that could have potentially developed in the study eyes over the follow-up period after PDT was calculated based on the following. In eyes that had no progression or less CNV leakage by TAP criteria (TAP 1999), the size of the potential DS was approximated by the size of the pre-treatment neovascular lesion. We believe this is appropriate because PDT is believed to cause occlusion of the vascular component of the neovascular lesion without affecting the fibrous component (Ghazi et al 2001). So although by TAP criteria (TAP 1999) the size (GLD) of the entire lesion and CNV leakage might angiographically decrease following PDT, the neovascular membrane, with its occluded vessels and fibrous matrix, anatomically persists with no regression. In eyes with progression following PDT, the size of the potential DS was approximated by the size of the post-treatment neovascular lesion at the last follow up visit.

\section{Results}

One hundred and forty eight eyes of 137 patients were treated with verteporfin-mediated PDT for SFCNV at the Department of Ophthalmology at the University of Virginia Health System over a period of 2 years. Careful review of the charts of these patients disclosed that 62 eyes of 60 patients satisfied the inclusion criteria of this study. The major reasons for exclusion included less than 6 months follow-up after the first PDT session, nonpredominantly classic SFCNV, and/or evidence of "RAP" (Yannuzzi et al 2001). The following is a report of the results obtained from analysis of the 62 eyes.

Sixty patients received PDT to only one eye and two patients had PDT performed to both eyes. Ninety-five percent (59/62) of the eyes had predominantly classic SFCNV secondary to AMD. The remaining 3 (5\%) were due to pathologic myopia ( 2 cases) and POHS (1 case). Twelve eyes (19\%) had recurrent SFCNV 
after having previously received laser photocoagulation for extrafoveal or juxtafoveal CNV. The follow up interval following the first PDT session ranged from 6 to 16 months (average 9 months) with $53 \%$ of the eyes followed for at least 9 months (equivalent of a maximum of 4 PDT sessions by TAP protocol). The number of treatment sessions over the period of follow-up ranged from 1 to 5 (average of 2.7 sessions). The average pre-treatment GLD was $2585 \mu \mathrm{m}$ (range: $400-4800 \mu \mathrm{m}$ ) and the average post-treatment GLD was $2103 \mu \mathrm{m}$ (range: $0-6800 \mu \mathrm{m}$ ).

At the last follow up visit following PDT, 40 eyes (64\%) had stable or decreased GLD of the lesion with no progression of leakage. Twenty-eight of 62 eyes (45\%) had no evidence of CNV with absence of leakage (Figure 1). Twenty-two eyes (36\%) had progression. Ten of these (45\%) had at least
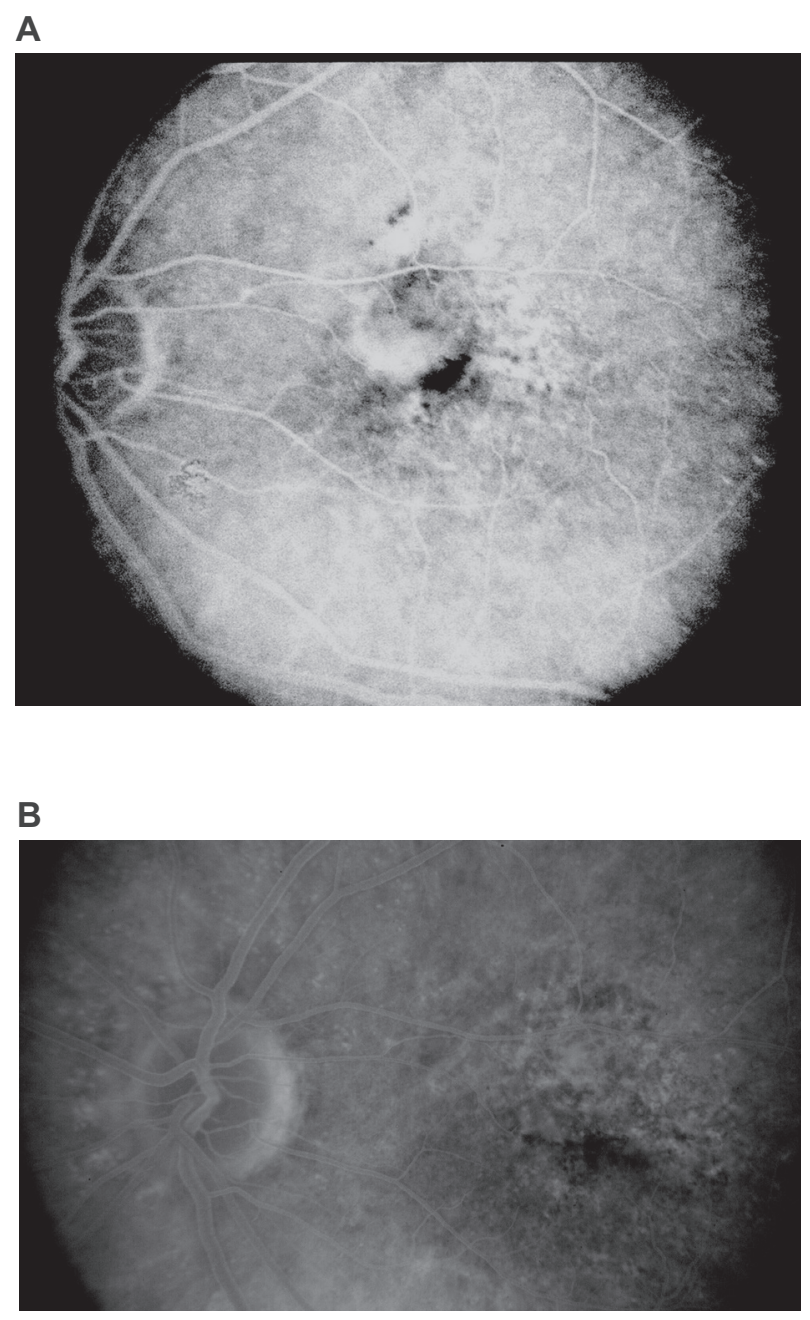

Figure I Pre-and post-treatment fluorescein angiogram (FA) of the left eye of a patient with AMD-related predominantly classic SFCNV.A) Pre-treatment, late phase frame. B) Ten months after two sessions of PDT, late phase frame. Note absence of CNV leakage in the post-treatment angiogram.

Abbreviations: AMD, age-related macular degeneration; CNV, choroidal neovascularization; PDT, photodynamic therapy; SFCNV, subfoveal choroidal neovascularization. doubling of the GLD of the neovascular lesion compared to pre-treatment. The latter could explain our observation that the average pre-treatment GLD $(2585 \mu \mathrm{m})$ was not significantly different from the average post-treatment GLD $(2103 \mu \mathrm{m})$, although $64 \%$ of the eyes had stable or decreased GLD. Three study eyes (5\%) developed a DS following PDT with an average scar size of $3200 \pm 1345 \mu \mathrm{m}$ (range: $2100-4700 \mu \mathrm{m})$ (Figure 2).

Fourteen patients $(23 \%)$ had already had disciform scarring in their fellow eye at the time of presentation, 6 of these (43\%) had previous laser therapy for CNV (Table 1). None of these eyes had previously received PDT. At last follow up visit, all but one of the 14 patients had a GLD of the neovascular lesion in the study eye smaller than the size of the scar in the fellow eye; and 7 of them (50\%), had no evidence of CNV with absence of leakage in the study eye (Table 1). The average size of the DS in these 14 fellow eyes was $5900 \pm 1866 \mu \mathrm{m}$ (range: $2200-9200 \mu \mathrm{m}$ ). The average size of the DS that developed in eyes with previous laser therapy (6/14) was $5500 \pm 2365 \mu \mathrm{m}$ (range: $2200-9200 \mu \mathrm{m}$ ) and that in eyes without previous intervention ( $8 / 14$; naturally occurring) was $6200 \pm 1493 \mu \mathrm{m}$ (range: $4000-8000 \mu \mathrm{m}$ ) (Figure 3; Table 2).

The average size of the DS that developed in 3 study eyes following PDT $(3200 \pm 1345 \mu \mathrm{m})$ was significantly smaller than that of the fellow eyes of the subgroup of 14 patients $(5900 \pm 1866 \mu \mathrm{m})$, and than that of the 8 of 14 eyes without previous intervention $(6200 \pm 1493 \mu \mathrm{m})$ (Tables 2, 3). It was not significantly smaller, however, than that of the 6 of 14 eyes that had disciform scaring with prior laser treatment $(5500 \pm 2365 \mu \mathrm{m})$ (Tables 2, 3).

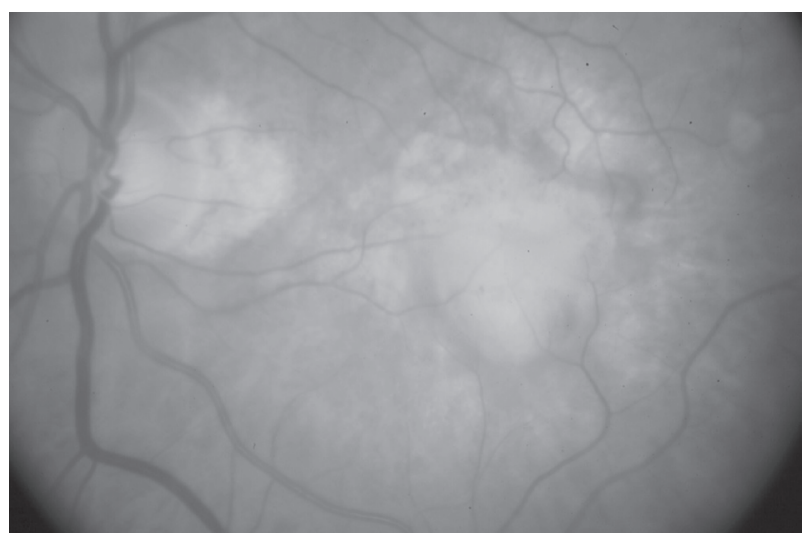

Figure 2 Disciform scar following PDT. Fundus picture of a patient with AMD-related SFCNV that evolved into a disciform scar following two sessions of PDT. This was the largest scar that developed following treatment (GLD: $4700 \mu \mathrm{m}$ ).

Abbreviations: AMD, age-related macular degeneration; GLD, greatest linear dimension; PDT, photodynamic therapy; SFCNV, subfoveal choroidal neovascularization. 
Table I Subgroup of I4 patients. GLD of the disciform scar in the fellow eyes and GLD of the neovascular lesion pre- and post-PDT in the study eyes

\begin{tabular}{lllll}
\hline Case number & GLD pre-PDT (micron) & GLD post PDT (micron) & $\begin{array}{l}\text { Size of disciform scar } \\
\text { in fellow eye (micron) }\end{array}$ & $\begin{array}{c}\text { Comments on } \\
\text { disciform scar }\end{array}$ \\
\hline 1 & & 0 & 6000 & Following laser \\
2 & 4400 & 4800 & 9200 & Following laser \\
3 & 1000 & 0 & 5200 & Following laser \\
4 & 2900 & 6000 & 6200 & \\
5 & 1800 & 4800 & 6400 & 7000 \\
6 & 3000 & 2800 & 8000 & Following laser \\
7 & 3000 & 0 & 4000 & Following laser \\
8 & 4000 & 3400 & 5600 & \\
9 & 1000 & 0 & 6400 & \\
10 & 4200 & 0 & 4000 & \\
11 & 400 & 0 & 4400 & \\
12 & 3600 & 0 & 2200 & \\
13 & 2000 & 4300 & 8000 & Following laser \\
\hline 4
\end{tabular}

Abbreviations: GLD, greatest linear dimension; PDT, photodynamic therapy.

The size of the DS that could have potentially developed following PDT was determined from the study eyes of the subgroup of 14 patients, and then also from the rest of the study eyes (45 eyes) excluding the 3 that developed DS. It was found to be $3607 \pm 1389 \mu \mathrm{m}$ and $3579 \pm 1473 \mu \mathrm{m}$, respectively (Table 2). In either case, it was significantly smaller than the average size of the scar in the fellow eyes of the subgroup of 14 patients $(5900 \pm 1866 \mu \mathrm{m})$, and of the 8 of 14 without previous intervention $(6200 \pm 1493 \mu \mathrm{m})$ (Tables 2,3). However, in both cases, it was not significantly smaller than that of the 6 of 14 eyes that developed the scar following prior laser treatment $(5500 \pm 2365 \mu \mathrm{m})$ (Tables 2, 3).

\section{Discussion}

Photodynamic therapy is now one of the first line treatment modalities used in the management of subfoveal CNV (VIP et al 2002). Its benefit in reducing vision loss has been proven in the case of AMD-related predominantly classic SFCNV (TAP 1999; Bressler 2001), AMD-related occult SFCNV (VIP 2001b; Bressler 2002), and SFCNV related to pathologic myopia (VIP 2001a). Some evidence also exists for a treatment benefit in other cases of SFCNV (Sickenberg et al 2000; Saperstein et al 2002; Busquets et al 2003). Although these clinical trials reported on the fluorescein angiographic outcome following treatment and provided evidence that PDT could limit the size and progression of the CNV lesion, the main emphasis was on the reduction in the risk of moderate and severe visual loss associated with PDT.
The effect of treatment on the size of the CNV lesion, particularly the evolution of the DS potentially associated with it, was less extensively assessed. Our study exclusively discusses this treatment benefit of PDT regardless of the final visual outcome, and supports the idea that the latter should not be the sole criterion by which treatment success is determined.

Over an average follow-up period of 9 months, we found that $64 \%$ of PDT treated eyes had stable or decreased GLD of the CNV lesion with no progression, $45 \%$ had no evidence of CNV with absence of leakage, and only $36 \%$ had progression at the last follow-up visit. These results compare favorably to those reported in the one-year follow up of the TAP trial (TAP 1999) where $19 \%$ of treated eyes had absence of leakage and $46 \%$ had progression. The explanation for this favorable outcome is two-fold. First, the majority of our study eyes had a lesion almost entirely composed of classic CNV (data not shown here). Although in a subgroup analysis in the TAP trial, lesion characteristics at baseline did not have a significant effect on the magnitude of treatment benefit on progression and final lesion size more than 6 MPS disc areas, only three broad classes of lesions were analyzed: those with at least $50 \%$, those with less than $50 \%$, and those with no classic CNV. Those with at least $50 \%$ classic $\mathrm{CNV}$ were not further subdivided. Our group of study eyes constitutes one subgroup of those with at least $50 \%$ classic $\mathrm{CNV}$ and probably the one with the most favorable response to PDT as far as post-treatment lesion size and progression are concerned. Second, a selection bias, by virtue of the 


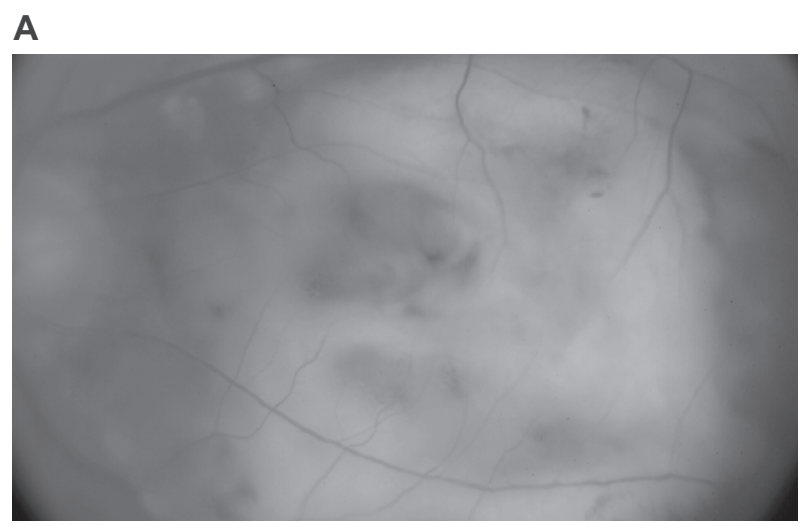

B

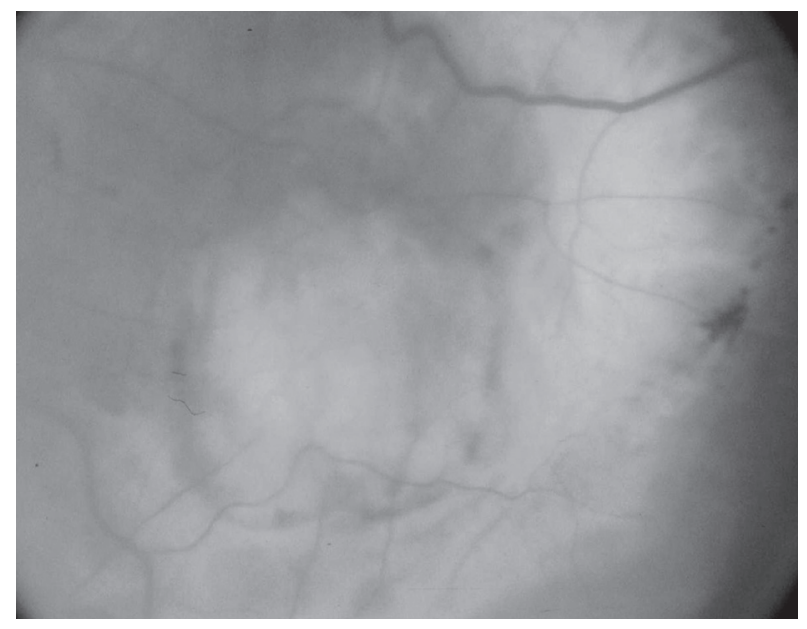

Figure 3 Disciform scars in 2 fellow eyes from the subgroup of 14 patients. A) Fundus picture of the largest scar that developed without previous intervention. B) Fundus picture of the largest scar that developed following previous laser treatment. This eye had received 2 sessions of laser photocoagulation for CNV.

Abbreviation: $\mathrm{CNV}$, choroidal neovascularization.

retrospective nature and inclusion criteria of the study, might be partially responsible. It is generally believed that patients with poor response to therapy are more likely to be noncompliant with treatment plans. Thus it is quite possible that the majority of eyes that had significant progression and poor visual outcome early after treatment failed to maintain the desired follow-up criteria of this study with either a short (less than 6 months) or an irregular (did not adhere to the TAP protocol) follow-up, leading to subsequent exclusion. The result of that would be the inadvertent selection of eyes with better response to PDT.

The effect of PDT on lesion size and progression emphasized in this manuscript might be very significant in determining the size of the DS and central scotoma that may ultimately develop in eyes with SFCNV. Disciform scarring is a frequent complication of neovascular maculopathy, particularly AMD (Green and Enger 1993). The evolution of a DS secondary to CNV has been described by Gass (1967a, 1967b) who noted that repeated episodes of hemorrhage and exudation from these abnormal vessels in the subretinal and/or subpigment epithelial space result in retinal pigment epithelium (RPE) metaplasia with vascular and fibroblastic in-growth from the choroid. The end result is a disciform maculopathy. Subsequent studies supported this theory (Teeters and Bird 1973; Green and Key 1977). Based on this, an intervention that limits the activity (bleeding and exudation) of CNV would be expected to favorably alter the evolution of the DS. PDT has been shown to cause endothelial cell damage and denudation of the vascular basement membrane with subsequent platelet aggregation, thrombus formation and vascular occlusion (Ghazi et al 2001). This effect, however, is short-lived because thrombus fragmentation and endothelial cell regeneration with re-population of the denuded basement membranes, result in re-perfusion of the occluded vessels following treatment (Ghazi et al 2001). This explains why repeated treatment sessions may be needed to maintain CNV non-perfusion. To understand the beneficial effect of PDT on the evolution of the DS despite its short-lived effect, it is important to examine what happens to the $\mathrm{CNV}$ in between treatment sessions.

At the vascular level, during the period that the neovessels are occluded following each treatment session, and before reperfusion and leakage re-occur, mild exudation and hemorrhage, if at all, takes place. At the cellular level, after each treatment, newly regenerated endothelial cells replace the treated necrotic cells. Evidence for this phenomenon can be observed experimentally as early as the third day following PDT and is complete by 2 weeks (Royster et al 1988). After the necrotic endothelial cells are regenerated, the following PDT session leads to their necrosis again, and the cycle continues with each treatment session. The newly regenerated, immature endothelial cells that replace the treated, mature cells might be less leaky. Evidence for this comes from previous ultrastructural studies which showed that development of endothelial fenestrations and loss of some of the surrounding pericytes are part of the maturation process of endothelial cells and capillaries lined by them (Ishibashi and Ryan 1992; Ishibashi et al 1995; Suzuki and Yoshida 1998). This may contribute to decreased exudation and hemorrhage in the early period following regeneration of treated capillaries and prior to their maturation. Therefore a logical implication would be that PDT could temporarily occlude active neovessels by virtue of thrombosis, and transform them into inactive vessels for at least a short period 
Table 2 Average size of disciform scars following PDT (actual and potential) and without PDT (with and without prior laser)

\begin{tabular}{lllllll}
\hline & $\begin{array}{l}\text { Disciform scar } \\
\text { in fellow eye } \\
\text { of subgroup } \\
\text { of I4 patients }\end{array}$ & $\begin{array}{l}\text { Disciform } \\
\text { scar in fellow } \\
\text { eyes with no } \\
\text { previous laser }\end{array}$ & $\begin{array}{l}\text { Disciform scar in } \\
\text { fellow eyes with } \\
\text { previous laser } \\
\text { treatment }\end{array}$ & $\begin{array}{l}\text { Disciform scar } \\
\text { in study eyes } \\
\text { following PDT }\end{array}$ & $\begin{array}{l}\text { Potential scar } \\
\text { post-PDT in study } \\
\text { eyes of subgroup } \\
\text { of I4 patients }\end{array}$ & $\begin{array}{l}\text { Potential scar } \\
\text { post-PDT in rest } \\
\text { of study eyes } \\
\text { excluding } 3 \text { eyes }\end{array}$ \\
\hline $\begin{array}{l}\text { Number of eyes } \\
\text { Average Size } \pm \text { SD }\end{array}$ & $5900 \pm 1866$ & 8 & 6 & 3 & 14 & 45 \\
(micron) & $6200 \pm 1493$ & $5500 \pm 2365$ & $3200 \pm 1345$ & $3607 \pm 1389$ & $3579 \pm 1473$ \\
\hline
\end{tabular}

Abbreviations: PDT, photodynamic therapy; SD, standard deviation.

following regeneration and reperfusion by virtue of capillary immaturity. Hence, less stimulus for RPE metaplasia and fibroblastic ingrowth as per Gass theory (Gass 1967a, 1967b), and a favorable influence on the evolution of the DS and central scotoma (Schmidt-Erfurth 1999).

The findings in our study support this hypothesis. The average size of the DS that developed in 3 study eyes following PDT ( $3200 \pm 1345 \mu \mathrm{m})$ was significantly smaller than the average size of the scar in the fellow eyes of the subgroup of 14 patients $(5900 \pm 1866 \mu \mathrm{m})$, and particularly the 8 of 14 without previous intervention $(6200 \pm 1493 \mu \mathrm{m})$ (Tables 2, 3). In addition, the average size of the potential DS derived from the study eyes of the subgroup of 14 patients $(3607 \pm 1389 \mu \mathrm{m})$ was significantly smaller than that of the scar in their fellow eyes $(5900 \pm 1866 \mu \mathrm{m})($ Tables 2, 3). One could argue that this comparison may be biased because patients with a DS and central scotoma in their fellow eye might seek care earlier due to the slightest disturbance of vision in their good eye secondary to increased awareness. To eliminate such bias, we also compared the average size of the DS in the fellow eyes of the subgroup of 14 patients $(5900 \pm 1866 \mu \mathrm{m})$ to that of the potential scar in the study eyes of the rest of the patients who do not have a DS in their fellow eye. The latter was found to be $3579 \pm 1437 \mu \mathrm{m}$ and was also significantly smaller (Tables 2,3 ). Therefore, the potential scar in both instances was significantly smaller than the DS in the fellow eyes of the 14 patients. This was also true when comparison was made with the $8 / 14$ fellow eyes that had a DS without previous intervention $(6200 \pm 1493 \mu \mathrm{m})$ (Tables 2, 3).

We also observed that there was no significant difference between the average size of the scar in the $6 / 14$ fellow eyes in which the scar developed following previous laser treatment $(5500 \pm 2365 \mu \mathrm{m})$ and that of the scar following PDT in the 3 study eyes that developed scarring ( $3200 \pm 1345 \mu \mathrm{m})$, the study eyes of the subgroup of 14 patients $(3607 \pm 1389 \mu \mathrm{m})$, or the study eyes of the rest of the patients $(3579 \pm 1473 \mu \mathrm{m})$, respectively (Tables 2,3 ). So it also appears that laser treatment, like PDT, might have a beneficial effect on the evolution of the DS. This, again, we believe is the result of the interruption of the active exudative phase of the CNV that leads to the development of disciform maculopathy.

One drawback of the subgroup analysis of our study is that the DS in the fellow eye of each of these 14 patients was already present at presentation. It was not prospectively observed to evolve; therefore, we could not tell with certainty whether the process started with SFCNV, or non-SFCNV with secondary subfoveal spread. Thus comparing disciform scars in these fellow eyes to actual and potential disciform scars secondary to documented SFCNV in the study eyes

Table 3 Comparison between the average size of the disciform scar following PDT (actual and potential) and that without PDT (with and without prior laser). Statistical analysis, p-value*

\begin{tabular}{llll}
\hline & $\begin{array}{l}\text { Disciform scar } \\
\text { in } 3 \text { study eyes } \\
\text { post-PDT }\end{array}$ & $\begin{array}{l}\text { Potential disciform scar } \\
\text { post-PDT in subgroup } \\
\text { of 14 study eyes }\end{array}$ & $\begin{array}{l}\text { Potential disciform scar } \\
\text { post-PDT in rest of study } \\
\text { eyes excluding } 3 \text { eyes }\end{array}$ \\
\hline $\begin{array}{l}\text { Disciform scar in subgroup of } \\
\text { I4 fellow eyes }\end{array}$ & 0.044 & 0.002 & 0.0001 \\
$\begin{array}{l}\text { Disciform scar in 8 fellow eyes } \\
\text { with no prior intervention }\end{array}$ & 0.033 & 0.002 & 0.0004 \\
$\begin{array}{l}\text { Disciform scar in 6 fellow eyes } \\
\text { following laser }\end{array}$ & 0.108 & 0.139 & 0.068 \\
\hline
\end{tabular}

Abbreviation: PDT, photodynamic therapy.

Note: *Student t-test. 
might be biased towards smaller scars in the study eyes. However, there is evidence that SFCNV is usually larger than non-SFCNV. Bressler and colleagues (1993) found that $66 \%$ of $\mathrm{CNV}$ involving the subfoveal region were bigger than $1500 \mu \mathrm{m}$ as opposed to $12 \%$ of CNV that did not. In addition, to our knowledge there is no evidence that the rate of CNV progression differs according to the location of the $\mathrm{CNV}$. Therefore the subsequent scar is expected to be larger for SFCNV which is the location of CNV that all our study eyes had at presentation. Our results suggest that PDT may alter this expectation with a beneficial effect on the evolution of the DS secondary to SFCNV. This appears to be related to the effect of PDT on the activity of the CNV as discussed above. Another drawback is that the DS that was already present in the fellow eye of these 14 patients at the time of initial examination might have developed over years. Thus, comparison with actual and potential disciform scars that developed or could have developed over the finite follow-up period of this study might be also biased. The best way to address this factor is by prospectively following a study group and a control group for a long period of time. However, the availability of treatment options such as PDT for SFCNV nowadays makes studies with a control group unfeasible. In addition, to our knowledge, these comparisons have not been performed in such prospective trials to date (TAP 1999; VIP 2001a, 2001b; Bressler 2001, 2002). A third drawback is that no measures of the central visual function other than visual acuity were performed on our patients. As has been demonstrated, central visual field function and scotoma characteristics as demonstrated by microperimetry may yield valuable information regarding final visual function and visual rehabilitation (Schmidt-Erfurth 2004).

This study shows evidence that the DS and potentially the subsequent central scotoma that might develop following PDT is expected to be smaller than that which develops naturally secondary to predominantly classic SFCNV. In microperimetric analysis studies of the central scotoma, PDT was associated with an improvement in the central visual field and reduction in scotoma size and intensity (Schmidt-Erfurth 1999, 2004). This might prove to be a very significant effect of PDT in neovascular maculopathy particularly in patients who fail the benefit of PDT in preventing visual loss. The literature has accumulating evidence that visual rehabilitation of patients with a smaller DS and central scotoma might be easier and more rewarding (Timberlake et al 1986, 1987; Whittaker et al 1988; Fletcher and Schuchard 1997; Schuchard and Fletcher 2000). This is because the size of the central scotoma is thought to be the most important factor in determining both the functional characteristics of the preferred retinal locus/loci (PRL(s)) that these patients develop for fixation, and the anatomic proximity of that locus/loci to the fovea (von Noorden and Mackensen 1962; Sunness et al 1996). Both factors may be significantly important in rehabilitating patients who lost central vision (von Noorden and Mackensen 1962; Fletcher and Schuchard 1997; Schuchard and Fletcher 2000). In recent studies, reading speed, macular sensitivity, macular electrophysiology, and fixation patterns correlated with the extent of macular impairment in choroidal neovascularization and appeared to improve following PDT (Ergun et al 2003; Midena et al 2004; Varano et al 2005; Yodoi et al 2007).

Ophthalmologists and researchers should not overlook the benefit of PDT emphasized in this paper. This important benefit has been probably underestimated and overshadowed because of the emphasis that ophthalmologists invest in the direction of final visual acuity outcome following treatment. Long-follow-up, controlled prospective studies with emphasis on the size of the DS and central scotoma, with utilization of microperimetry with scanning laser ophthalmoscopy could demonstrate the value of PDT in limiting the size of the eventual DS and central scotoma in patients with predominantly classic SFCNV. Also they could possibly determine any beneficial effect of PDT in the rehabilitation process of treated patients. However, prospectively following a control group with SFCNV might not be feasible nowadays due to the availability of treatment options such as PDT. Re-evaluating patients enrolled in previous clinical trials (TAP 1999; VIP 2001a, 2001b; Bressler 2001, 2002) is one potential way for testing our hypothesis.

\section{Disclosure}

None of the authors have any proprietary or conflict of interest related to the manuscript.

\section{References}

Bressler NM, Bressler SB. 2000. Photodynamic therapy with verteporfin (Visudyne): impact on ophthalmology and visual sciences. Invest Ophthalmol Vis Sci, 41:624-8.

Bressler NM. 2001. Photodynamic therapy of subfoveal choroidal neovascularization in age-related macular degeneration with verteporfin: two-year results of 2 randomized clinical trials - TAP Report 2. Arch Ophthalmol, 119:198-207.

Bressler NM. 2002. Verteporfin therapy of subfoveal choroidal neovascularization in age-related macular degeneration: two-year results of a randomized clinical trial including lesions with occult with no classic choroidal neovascularization - verteporfin in photodynamic therapy report 2. Am J Ophthalmol, 133:168-9. 
Bressler SB, Bressler NM, Fine SL, et al. 1983. Subfoveal neovascular membranes in senile macular degeneration. Relationship between membrane size and visual prognosis. Retina, 3:7-11.

Busquets MA, Shah GK, Wickens J, et al. 2003. Ocular photodynamic therapy with verteporfin for choroidal neovascularization secondary to ocular histoplasmosis syndrome. Retina, 23:299-306.

Ergun E, Maar N, Radner W, et al. 2003. Scotoma size and reading speed in patients with subfoveal occult choroidal neovascularization in agerelated macular degeneration. Ophthalmology, 110:65-9.

Ferris III FL, Fine SL, Hyman L. 1984. Age-related macular degeneration and blindness due to neovascular maculopathy. Arch Ophthalmol, 102:1640-2.

Fletcher DC, Schuchard RA. 1997. Preferred retinal loci: relationship to macular scotomas in a low-vision population. Ophthalmology, 104:632-8.

Gass JDM. 1967a. Pathogenesis of disciform detachment of the neuroepithelium. III. Senile disciform macular degeneration. Am J Ophthalmol, 63:617-44.

Gass JDM. 1967b. Pathogenesis of disciform detachment of the neuroepithelium. IV. Fluorescein angiographic study of senile disciform macular degeneration. Am J Ophthalmol, 63:645-59.

Ghazi NG, Jabbour NM, De La Cruz ZC, et al. 2001. Clinicopathologic studies of age-related macular degeneration with classic subfoveal choroidal neovascularization treated with photodynamic therapy. Retina, 21:478-86.

Green WR, Enger C. 1993. Age-related macular degeneration histopathologic studies. The 1992 Lorenz E. Zimmerman Lecture. Ophthalmology, 100:1519-35.

Green WR, Key 3rd SN. 1977. Senile macular degeneration: a histopathologic study. Trans Am Ophthalmol Soc, 75:180-254.

Ishibashi T, Inomata H, Sakamoto T, et al. 1995. Pericytes of newly formed vessels in experimental subretinal neovascularization. Arch Ophthalmol, 113:227-31.

Ishibashi T, Ryan SJ. 1992. Maturation of newly-formed subretinal vessels. EXS, 61:59-63.

Klein R, Wang Q, Klein BE, et al. 1995. The relationship of age-related maculopathy, cataract, and glaucoma to visual acuity. Invest Ophthalmol Vis Sci, 36:182-91.

[MPSG] Macular Photocoagulation Study Group. 1993. Laser photocoagulation of subfoveal neovascular lesions of age-related macular degeneration. Arch Ophthalmol, 111:1200-9.

Margherio RR, Margherio AR, DeSantis ME. 2000. Laser treatment with verteporfin therapy and its potential impact on retinal practices. Retina, 20:325-30.

Michels S, Barbazetto I, Schmidt-Erfurth U. 2000. Choroidal changes after photodynamic therapy (PDT). A two year follow-up study of 38 patients. Klin Monatsbl Augenheilkd, 217:94-9.

Midena E, Radin PP, Pilotto E, et al. 2004. Fixation pattern and macular sensitivity in eyes with subfoveal choroidal neovascularization secondary to age-related macular degeneration. A microperimetry study. Semin Ophthalmol, 19:55-61.

Olk RJ, Burgess DB, McCormick PA. 1984. Subfoveal and juxtafoveal subretinal neovascularization in the presumed ocular histoplasmosis syndrome. Visual prognosis. Ophthalmology, 91:1592-602.

Royster AJ, Nanda SK, Hatchell DL, et al. 1988. Photochemical initiation of thrombosis. Fluorescein angiographic, histologic, and ultrastructural alterations in the choroid, retinal pigment epithelium and retina. Arch Ophthalmol, 106:1608-14.

Saperstein DA, Rosenfeld PJ, Bressler NM, et al; Verteporfin in Ocular Histoplasmosis (VOH) Study Group. 2002. Photodynamic therapy of subfoveal choroidal neovascularization with verteporfin in the ocular histoplasmosis syndrome: one-year results of an uncontrolled, prospective case series. Ophthalmology, 109:1499-505.

Schmidt-Erfurth U. 1999. Indocyanine green angiography and retinal sensitivity after photodynamic therapy of subfoveal choroidal neovascularization. Semin Ophthalmol, 14:35-44.
Schmidt-Erfurth UM, Elsner H, Terai N, et al. 2004. Effects of verteporfin therapy on central visual field function. Ophthalmology, 111:931-9.

Schuchard RA, Fletcher DC. 2000. Preferred retinal locus and the scanning laser ophthalmoscope. In: Albert DM, Jacobiec FA (eds). Principles and practice of ophthalmology. St. Lowis: WB Saunders Company, 2nd Edition, Vol. 2, Ch. 395:5438-43.

Sickenberg M, Schmidt-Erfurth U, Miller JW, et al. 2000. A preliminary study of photodynamic therapy using verteporfin for choroidal neovascularization in pathologic myopia, ocular histoplasmosis syndrome, angioid streaks and idiopathic causes. Arch Ophthalmol, 118:327-36

Soubrane G, Coscas GJ. 2001. Choroidal neovascularization in degenerative myopia. In: Ryan SJ, Wilkinson CP (eds). Retina. St. Lowis: Mosby, 3rd Edition, Vol. 2, Ch. 67:1136-52.

Sunness JS, Applegate CA, Haselwood D, et al. 1996. Fixation patterns and reading rates in eyes with central scotomas from advanced atrophic age-related macular degeneration and Stargardt disease. Ophthalmology, 103:1458-66.

Suzuki H, Yoshida K. 1998. Electron microscopic study of capillary in the human renal cell carcinoma. Nippon Hinyokika Gakkai Zasshi, 89:14-22.

Teeters VW, Bird AC. 1973. A clinical study of the vascularity of senile disciform macular degeneration. Am J Ophthalmol, 75:53-65.

Timberlake GT, Mainster MA, Peli E, et al. 1986. Reading with a macular scotoma. I. Retinal location of scotoma and fixation area. Invest Ophthalmol Vis Sci, 27:1137-47.

Timberlake GT, Peli E, Essock EA, et al. 1987. Reading with a macular scotoma. II. Retinal locus for scanning text. Invest Ophthalmol Vis Sci, 28:1268-74.

[TAP] Treatment of Age-Related Macular Degeneration With Photodynamic Therapy Study Group. 1999. Photodynamic therapy of subfoveal choroidal neovascularization in age-related macular degeneration with verteporfin: one-year results of 2 randomized clinical trials - TAP Report 1. Arch Ophthalmol, 117:1329-45.

[VIP] Verteporfin in Photodynamic Therapy Study Group. 2001a. Photodynamic therapy of subfoveal choroidal neovascularization in pathologic myopia with verteporfin. 1-year results of a randomized clinical trial - VIP report no. 1. Ophthalmology, 108:841-52.

[VIP] Verteporfin In Photodynamic Therapy Study Group. 2001b. Verteporfin therapy of subfoveal choroidal neovascularization in age-related macular degeneration: two-year results of a randomized clinical trial including lesions with occult with no classic choroidal neovascularization - verteporfin in photodynamic therapy (VIP) report 2. Am J Ophthalmol, 131:541-60.

[VIP] Verteporfin Roundtable 2000 and 2001 Participants, Treatment of Age-Related Macular Degeneration With Photodynamic Therapy (TAP) Study Group Principal Investigators, Verteporfin In Photodynamic Therapy (VIP) Study Group Principal Investigators. 2002. Guidelines for using verteporfin (Visudyne ${ }^{\circledR}$ ) in photodynamic therapy to treat choroidal neovascularization secondary to age-related macular degeneration and other causes. Retina, 22:6-18.

Varano M, Parisi V, Tedeschi M, et al. 2005. Macular function after PDT in myopic maculopathy: psychophysical and electrophysiological evaluation. Invest Ophthalmol Vis Sci, 46:1453-62.

von Noorden GK, Mackensen G. 1962. Phenomenology of eccentric fixation. Am J Ophthalmol, 53:642-61.

Whittaker SG, Budd J, Cummings RW. 1988. Eccentric fixation with macular scotoma. Invest Ophthalmol Vis Sci, 29:268-78.

Yannuzzi LA, Negrao S, Iida T, et al. 2001. Retinal angiomatous proliferation in age-related macular degeneration. Retina, 21:416-34.

Yodoi Y, Tsujikawa A, Kameda T, et al. 2007. Central retinal sensitivity measured with the micro perimeter 1 after photodynamic therapy for polypoidal choroidal vasculopathy. Am J Ophthalmol, 143:984-94. 